gave a conflicting history. However, his response to treatment and the subsequent course of his condition rule out a simple drug-induced psychosis, and our favoured diagnosis is paranoid schizophrenia.

Fish's Clinical Psychopathology (Hamilton, 1974) asserts that delusional ideas, in contrast to overvalued ideas, are not commonly acted upon. Our case, together with those of Drs Shubsachs \& Young and Drs de Pauw \& Szulecka (Journal, January 1988 , 152, 91-97), suggests that, in delusional misidentification syndromes at least, this assertion needs to be treated with as much caution as the patients!

A. R. TOMison

Butler Clinic W. M. DONOVAN

Langdon Hospital

Exeter Road

Dawlish EX7 0NR

Reference

Hamilton, M. (1974) Fish's Clinical Psychopathology. Bristol: John Wright \& Sons.

\section{Response to Biological Treatment}

SIR: As a clinical biochemist working in psychiatry, the article by Myers (Journal, May 1988, 152, 657659) on the prediction of response of depressed patients to treatment provokes me to a comment. Not on the design or results of the study, which are interesting enough, but on a more than purely semantic problem.

Because biological psychiatry studies biochemistry, physiology, anatomy, etc. in relation to psychiatric disorders, and because pharmacological findings do considerably contribute to the increase of knowledge in biological psychiatry, this does not imply that applied psychopharmacology, i.e. drug treatment, in psychiatry is a biological treatment.

The generally accepted meaning of the word 'biological' implies a relation to the science of life or to life processes and phenomena. I sincerely hope we may prevent a deterioration of the sense of the term 'biological' analogous to that of the term 'chemical' to mean synthetic or artificial. Calling the use of antidepressant drugs a 'biological' treatment is more than confusing. Why not 'drug' or 'pharmacological' treatment? In this respect it may even be maintained that psychotherapy, for example, is a more biological treatment than the use of drugs.

G. M. J. VAN KEMPEN

Psychiatric Hospital Endegeest

POB 1250

2340 BG Oegstgeest

The Netherlands

\section{The Pathogenesis of Depersonalisation}

SIR: Before Professor Cohen's suggestion of a causal link between hyperventilation and depersonalisation becomes universally "received knowledge" (Journal, April 1988, 152, 578), it would be helpful to know what were his criteria for diagnosing hyperventilation, and whether the traditional brown paper bag is effective in depersonalisation.

In my experience, when the two conditions co-exist the anxiety is the result of the experience of depersonalisation and the latter is often of such abrupt onset that it would be hard to explain it in biochemical terms related to overbreathing. Moreover, a positive understanding of the personal meaning of the symptom can often be acquired through patient self-monitoring (Ryle, 1982), as the following two cases illustrate.

Case reports: (i) A young man experienced intense depersonalisation at the railway station after a visit to his mother. Since his father's death 5 years previously he had felt overinvolved with his mother, and visits were marked by a mutual exacerbation of depression and psychosomatic symptoms. His attack had generated panic and the fear of going mad. Self-monitoring of subsequent attacks revealed that all occurred as an immediate consequence of a thought, event, or another's remark implying the possibility that he might be sexually potent, or, on one occasion, followed him seeing himself in the barber's mirror and thinking "Macbeth, Lear, Oedipus". In psychoanalytic terms, therefore, his depersonalisation (aptly named) removed him from the possibility of profiting from his 'oedipal victory' over his father.

(ii) A 25-year-old man had suffered for 2 years from severe depression with numerous suicide attempts. Over this period he had been increasingly disabled by intense and frequent depersonalisation experiences. His older sibling had been a furious child and had become schizophrenic during adolescence. $\mathrm{He}$, in contrast, had been a compliant, high-achieving but emotionally cut-off person. Selfmonitoring of his depersonalisation showed it to be provoked by feeling controlled or misunderstood, and he was able to recognise a flash of anger prior to the onset of the symptom. In this case also, the symptom can be seen to prevent the patient becoming the kind of person who, for reasons of his history, was unacceptable.

Depersonalisation should be considered in terms of its personal meaning to the sufferer; surely Professor Cohen is not suggesting that all disorders of perception have an organic base?

York Clinic and Munro Clinic

ANTHONY RYLE Guy's Hospital

London Bridge SE1 9RT

\section{Reference}

RYLE, A. (1982) Psychotherapy: A Cognitive Integration of Theory and Practice. London: Academic Press. 